\title{
An updated picture of the mental health needs of male and female prisoners in the UK: prevalence, comorbidity, and gender differences
}

\author{
Nichola Tyler ${ }^{1,2} \oplus \cdot$ Helen L. Miles ${ }^{1,2,3} \cdot$ Bessey Karadag $^{2} \cdot$ Gemma Rogers $^{2}$
}

Received: 30 August 2018 / Accepted: 9 March 2019 / Published online: 22 March 2019

(c) The Author(s) 2019

\begin{abstract}
Purpose Epidemiological data on the mental health needs of prisoners are essential for the organisation, planning, and delivery of services for this population as well as for informing policy and practice. Recent reports by the National Audit Office and NICE call for new research to provide an updated picture of the mental health needs of men and women in prison in the UK. This study aimed to measure the prevalence and comorbidity of mental health needs across a representative sample of both men and women across 13 prisons in one UK region.

Method Participants completed a standardised battery of psychometric assessments which screened for a range of mental health difficulties including: mental disorders, personality disorder, and substance misuse.

Results 469 participants were included in the final sample (338 males, 131 females). A high number of participants reported having had previous contact with mental health services and/or a pre-existing diagnosis of a mental disorder. High rates of current mental disorder were detected across the range of disorders screened for. Levels of comorbidity were also high, with nearly half of participants screening positive for two or more types of mental disorder. Gender differences were noted in terms of previous contact with mental health services, having a pre-existing diagnosis, prevalence of current mental disorder, and levels of comorbidity; with women reporting higher rates than men.

Conclusions Rates of pre-existing and current mental illness continue to be high amongst prisoners. Women report significantly higher levels of mental health need compared to men.
\end{abstract}

Keywords Prisons $\cdot$ Forensic mental health $\cdot$ Offenders $\cdot$ Prisoners $\cdot$ Prison healthcare

\section{Introduction}

Research has consistently shown that rates of mental disorder are higher amongst people in prison than in the general population [1]. However, a recent report by the National

The views expressed are those of the authors and do not necessarily reflect those of the NHS, Her Majesty's Prison and Probation Service, or the Ministry of Justice.

Nichola Tyler

N.F.Tyler@kent.ac.uk

1 Centre of Research and Education in Forensic Psychology, School of Psychology, University of Kent, Canterbury CT2 7NP, UK

2 Forensic and Specialist Care Group, Kent and Medway NHS and Social Care Partnership Trust, Greenacres, Dartford, Kent DA2 6PB, UK

3 Institute of Psychiatry, Psychology and Neuroscience, Kings College London, London, UK
Audit Office [2] identified a deficit in the provision of mental health services within UK prisons and a lack of parity of care with community services. Both the National Audit Office Report [2], and the recently updated NICE guidelines on mental health in the criminal justice system [3], identified a need for new epidemiological research into the mental health of individuals in the criminal justice system; to examine how the mental and social functioning of individuals in prison in the UK has changed since the last major epidemiological study conducted in 1997 (The Office of National Statistics [ONS] Survey of Psychiatric Morbidity among Prisoners in England and Wales) [4]. Since the ONS survey, UK-based research in this area has predominantly focused on screening for the prevalence of individual disorders (or clusters of disorders) across a small number of establishments, and those studies which have conducted more in-depth screenings of multiple disorders collected data over 10 years ago [4-7]. In the last 20 years there have been a number of significant changes to the prison system (e.g., increase in 
population, reduction in staffing, and a change of function for some establishments) as well as mental health and social care practice in this context. It is, therefore, important to have an updated picture of the mental health needs of those in prison to plan and inform effective provision of services in these settings. This study examines (a) the current prevalence of mental disorder amongst male and female prisoners sampled from all establishments in one region of the UK, (b) the current level of comorbidity of disorders amongst this population, and (c) any gender differences in these areas.

\section{Method}

\section{Ethics}

Ethical approval for the study was received from the University of Kent Research Ethics Committee (Ref: 201614727338303972), NHS Health Research Authority (REC Ref: 16/WA/0316) and Her Majesty's Prison and Probation Service National Research Committee (NRC Ref: 16-323).

\section{Study design and setting}

A cross sectional survey was conducted across all 13 prisons ( 9 male and 4 female) in one region in the South of England; no prisons in the region were excluded from the research. Prisoners residing in both open and closed conditions were represented in the sample ( 3 open, 11 closed), as well as a range of different risk category of establishment (4 Category B, 3 Category C, 2 Category D, 4 women's prisons), and individuals at different points in the custodial pathway (e.g., remand and sentenced, range of sentence lengths). Of those establishments where data were collected from, eight held exclusively sentenced prisoners (6 male and 3 female) with the remaining four prisons housing both sentenced and remand prisoners.

\section{Participants}

A total of 766 potential participants were initially selected at random, using a sequential system, from the local P-NOMIS database across the 13 participating establishments. Sampling was conducted on a per prison basis with targeted proportions of approximately 5\% of the population at each male establishment and approximately $10 \%$ of the population at each female establishment recruited. A larger sample target was selected for female prisons to ensure a representative sample size was collected from these establishments since these are smaller in capacity

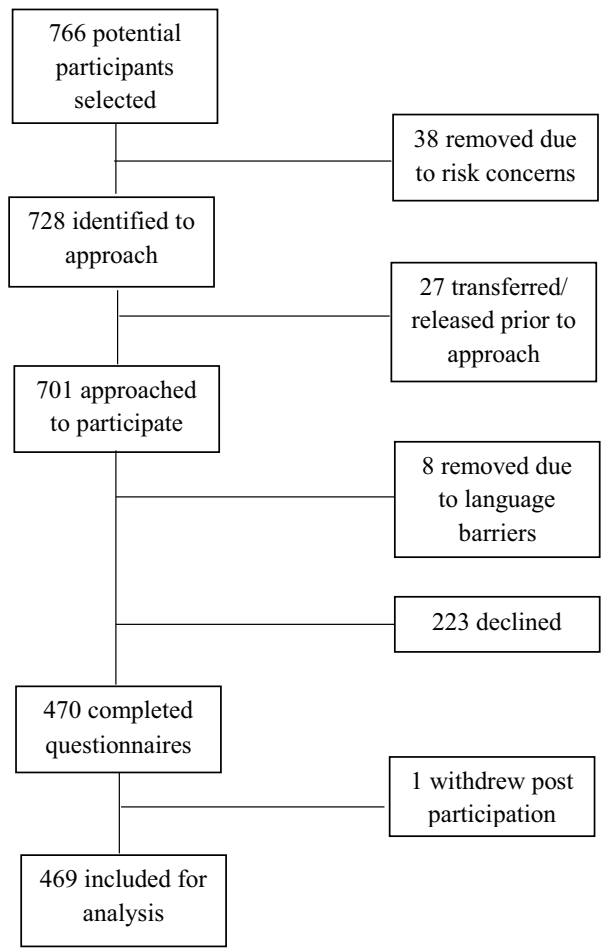

Fig. 1 Flow of participants through the study

than the male prisons in the region. Thirty-eight potential participants were removed following initial selection due to risk concerns, leaving 728 individuals considered suitable to approach. A further 27 potential participants were lost to recruitment due to being transferred or released prior to being approached by the research team. Of the 701 potential participants who were able to be invited to the research, 8 were removed due to significant language barriers preventing informed consent and 223 declined to participate in the study. Thus, a total of 470 participants completed an assessment session. One participant withdrew from the study following participation leaving 469 in the final sample (see Fig. 1 for an overview of participants' flow through the study). The overall response rate for the study was $68.2 \%$.

\section{Measures}

\section{Demographic information}

A questionnaire was developed to capture demographic information, including age, ethnicity, highest level of qualification, sentence status (e.g., remand or sentenced), sentence length, index offence, prior contact with mental health services, lifetime diagnosis of a mental disorder, and current contact with Mental Health Inreach. 


\section{Screening measures}

A battery of well-established self-report screening tools, commonly used in clinical and forensic settings, were used to screen for a range of mental health issues including personality disorder, clinical syndromes (e.g., anxiety, somatoform, mood disorders, ${ }^{1}$ PTSD, and psychotic disorders ${ }^{2}$ ), drug and alcohol dependence, eating disorders, and risk of suicidal behaviours. Screening tools were selected for use in the current study as they are frequently used in research and practice with prison samples [4, 8-12].

Traits indicative of personality disorder and clinical syndromes were screened for using the Millon Clinical Multiaxial Inventory Third Edition (MCMI-III) [13]. Drug dependence in the year prior to prison was screened for using the Severity of Dependence Scale (SDS) [14]; harmful alcohol consumption in the year prior to prison was screened for using a short-form of the Alcohol Use Disorders Identification Test (AUDIT-PC) [15]. Risk of suicidal behaviour was screened for using the Suicide Behaviours QuestionnaireRevised (SBQR) [16, 17]. Eating disorders were screened for using the SCOFF [18]. All measures were scored using the relevant scoring guidance. Participants were considered to have screened positive for a particular disorder if their total score was above the defined established clinical cut off score for the measure/subscale, indicating levels of clinical symptomatology to suggest presence of that disorder. Total scores were then dichotomised into either presence or absence of each disorder (i.e., above or below the cut-off).

\section{Procedure}

This study formed part of a more in-depth mental health needs assessment across the participating prisons, consisting of a larger battery of screening measures. All researchers had undertaken postgraduate level training in psychology and had received specific training in the administration of each of the screening tools from a Consultant Clinical and Forensic Psychologist, prior to data collection. Initial assessments with all researchers were observed by the first author and further spot checks conducted throughout the project for quality assurance and standardisation purposes. Researchers received regular supervision throughout data collection from both the first and second author.

Informed consent was obtained from all participants prior to partaking in the assessment session. Screening measures were completed with participants at a single time point between February and September 2017. All screening measures were read aloud to participants by a researcher to

\footnotetext{
${ }^{1}$ Major depression, bipolar, dysthymia.

2 Thought disorder, delusional disorder.
}

ensure adequate comprehension and understanding. Each assessment session was conducted either on a one-to-one or two-to-one basis (on the advice of prison staff based upon operational or risk-related issues) with a member(s) of the research team, and lasted on average 75-90 min in length. Measures were presented in a randomised order to counteract any ordering effects or respondent fatigue with the exception of the demographic questionnaire which was always administered first.

\section{Statistical analysis}

For the purpose of analysis, measures were collapsed into categories of diagnostically related disorders (e.g., anxiety disorders, personality disorders, mood disorders, ${ }^{3}$ eating disorders, substance use disorders, psychotic disorders, ${ }^{4}$ risk of suicidal behaviour, somatoform, post-traumatic stress disorder). Prevalence rates were then calculated based upon the number of participants who scored above the clinical cut-off on at least one of the measures within each category of disorder (e.g., scored above the clinical cut off for any mood disorder).

Descriptive statistics were computed to summarise participant's demographic, psychiatric and offence characteristics and to calculate the point-prevalence of each category of mental disorder (i.e., the percentage of participants who screened above the clinical cut off score for each type of disorder). Pearson's Chi square was used to examine gender differences between prevalence rates across previous contact with mental health services and current mental disorder. How well current mental health needs were being met was examined using Crosstabs and Chi square to calculate the proportion of male and female participants who screened positive for both a current mental disorder and current engagement with prison mental health services.

\section{Results}

\section{Characteristics of the study sample}

The sample consisted of 469 male and female prisoners (males 338, females 131). Participants' ages ranged from 18 to 80 years $(M=35.95$, SD 11.68; Mdn 34.00, IQR 26-43). The majority of participants were sentenced $(90.2 \%)$, with the average sentence length reported at 260.45 weeks (range 1 to 1300 weeks, Mdn 184, IQR 52-386) (see Table 1 for full details of participant characteristics). The characteristics of the sample are broadly in line with that of the current

\footnotetext{
${ }^{3}$ Major depression, bipolar, dysthymia.

4 Thought disorder, delusional disorder.
} 
Table 1 Demographics for the sample

\begin{tabular}{|c|c|c|c|}
\hline Characteristic & Total sample, $\%(n)$ & Males, \% (n) & Females, \% (n) \\
\hline \multicolumn{4}{|l|}{ Age } \\
\hline $18-20$ & $4.1(19)$ & $4.1(14)$ & $3.8(5)$ \\
\hline $21-30$ & $34.8(163)$ & $36.7(124)$ & $29.8(39)$ \\
\hline $31-40$ & $28.6(134)$ & $27.5(93)$ & $31.3(41)$ \\
\hline $41-50$ & $19.4(91)$ & $18.0(61)$ & $22.9(30)$ \\
\hline $51-60$ & $9.6(45)$ & $9.8(33)$ & $9.2(12)$ \\
\hline 61 and above & $3.6(17)$ & $3.8(13)$ & $3.1(4)$ \\
\hline \multicolumn{4}{|l|}{ Ethnicity $^{\mathrm{a}}$} \\
\hline Asian & $6.0(28)$ & $6.6(22)$ & $4.6(6)$ \\
\hline Black Caribbean & $6.8(32)$ & $7.7(26)$ & $4.6(6)$ \\
\hline Black African & $7.2(34)$ & $7.1(24)$ & $7.6(10)$ \\
\hline Mixed race & $7.2(34)$ & $5.6(19)$ & $11.5(15)$ \\
\hline White UK/Irish & $58.0(272)$ & $57.1(193)$ & $60.3(79)$ \\
\hline White other & $8.7(41)$ & $10.0(34)$ & $5.3(7)$ \\
\hline Other & $5.7(27)$ & $5.6(19)$ & $6.2(8)$ \\
\hline \multicolumn{4}{|l|}{ Qualifications $^{\mathrm{b}}$} \\
\hline No qualifications & $27.9(131)$ & $29.3(99)$ & $24.4(32)$ \\
\hline Fifth form level & $34.8(163)$ & 34.9 (118) & $34.4(45)$ \\
\hline Sixth form level & $26.6(125)$ & $26.6(90)$ & $26.7(35)$ \\
\hline Bachelor degree or above & $10.2(48)$ & $8.9(30)$ & $13.8(18)$ \\
\hline \multicolumn{4}{|l|}{ Index offence ${ }^{c}$} \\
\hline Violent & $33.9(159)$ & $33.8(114)$ & $34.4(45)$ \\
\hline Non-violent & $5.5(26)$ & $5.6(19)$ & $5.3(7)$ \\
\hline Property damage/arson & $1.7(8)$ & $0.6(2)$ & $4.6(6)$ \\
\hline Substance related offences & $23.2(109)$ & $24.0(81)$ & $21.4(28)$ \\
\hline Acquisitive/fraud & $24.9(117)$ & $23.9(81)$ & $27.5(36)$ \\
\hline Terrorism & $0.6(3)$ & $0.9(3)$ & $0.0(0)$ \\
\hline Sexual offences & $7.2(34)$ & $9.5(32)$ & $1.5(2)$ \\
\hline Civil offences & $0.4(2)$ & $0.3(1)$ & $0.8(1)$ \\
\hline Immigration & $0.2(1)$ & $0.0(0)$ & $0.8(1)$ \\
\hline Prison related offences & $0.2(1)$ & $0.0(0)$ & $0.8(1)$ \\
\hline \multicolumn{4}{|l|}{ Sentence } \\
\hline Remand & $9.8(46)$ & $8.9(30)$ & $12.2(16)$ \\
\hline Sentenced & $90.2(423)$ & $91.1(308)$ & $87.8(115)$ \\
\hline \multicolumn{4}{|l|}{ Psychiatric history } \\
\hline $\begin{array}{l}\text { Previous contact mental health } \\
\text { services }\end{array}$ & $48.8(229)$ & 43.5 (147) & $62.6(82)$ \\
\hline
\end{tabular}

${ }^{\mathrm{a}}$ One male participant did not disclose his ethnicity

${ }^{\mathrm{b}}$ Two participants did not disclose/did not know their highest qualification

${ }^{\mathrm{c}}$ Nine participants declined to disclose their Index Offence general prison population in terms of age, ethnicity, average sentence length, and proportion on remand $[19,20]$.

\section{Prior engagement with psychiatric services}

Of the total sample, $48.8 \%(n=229)$ of participants reported having had previous contact with mental services either in prison or in the community. Further, $42.4 \%(n=199)$ of participants reported having previously received a diagnosis of a mental illness. Females were significantly more likely to report having had previous contact with mental health services than males, $\chi^{2}(1, N=469)=13.79, p=0.000$, and were also significantly more likely to report having previously received a diagnosis of a mental disorder, $\chi^{2}(1$, $N=465)=29.21, p=0.000$. In terms of diagnoses, females were significantly more likely than males to report having previously received a diagnosis of a personality disorder, mood disorder, obsessive compulsive disorder or an eating 
Table 2 Lifetime diagnoses for participants by gender

\begin{tabular}{lccclll}
\hline Diagnoses & $\begin{array}{l}\text { Overall sample, } \\
\%(n), N=199\end{array}$ & $\begin{array}{l}\text { Males, } \\
\%(n), \\
N=117\end{array}$ & $\begin{array}{l}\text { Females, } \% \\
(n), N=82\end{array}$ & $\chi^{2}$ & $p$ & Effect size \\
\hline Personality disorder & $26.5(51)$ & $20.0(22)$ & $35.4(29)$ & 6.94 & $0.008^{*}$ & 0.187 \\
Anxiety disorder & $27.1(52)$ & $26.3(29)$ & $28.0(23)$ & 0.27 & 0.606 & 0.037 \\
Mood disorder & $58.8(117)$ & $51.8(57)$ & $73.2(60)$ & 11.90 & $0.001^{*}$ & 0.245 \\
PTSD & $19.8(38)$ & $20.9(23)$ & $18.3(15)$ & 0.06 & 0.809 & 0.017 \\
Psychotic disorder & $9.9(19)$ & $13.6(15)$ & $4.9(4)$ & - & $0.085^{\mathrm{a}}$ & 0.133 \\
Brain injury & $0.5(1)$ & $0.9(1)$ & $0.0(0)$ & - & $1.00^{\mathrm{a}}$ & 0.401 \\
Autism spectrum conditions & $3.7(7)$ & $5.4(6)$ & $1.2(1)$ & - & $0.243^{\mathrm{a}}$ & 0.104 \\
Learning disability & $1.0(2)$ & $1.8(2)$ & $0.0(0)$ & - & $0.513^{\mathrm{a}}$ & 0.084 \\
ADHD & $14.6(28)$ & $20.0(22)$ & $7.3(6)$ & 5.26 & $0.022^{*}$ & 0.163 \\
Obsessive compulsive disorder & $5.7(11)$ & $1.8(2)$ & $11.0(9)$ & - & $0.009^{* \mathrm{a}}$ & 0.200 \\
Dementia & $1.0(2)$ & $0.9(1)$ & $1.2(1)$ & - & $1.00^{\mathrm{a}}$ & 0.018 \\
Eating disorder & $3.6(7)$ & $0.0(0)$ & $8.5(7)$ & - & $0.002^{* \mathrm{a}}$ & 0.228 \\
\hline
\end{tabular}

Participants may have held more than one diagnosed disorder

*Significant $p<0.05$

${ }^{a}$ Fisher's exact used due to low cell count

Table 3 Prevalence of disorders screened positive for on measures

\begin{tabular}{|c|c|c|c|c|c|c|}
\hline Disorder & Total sample, \% (n) & Males, \% (n) & Females, \% (n) & $\chi^{2}$ & $p$ & Effect size \\
\hline Any Personality Disorder & $54.8(257)$ & 49.4 (167) & $68.7(90)$ & 20.62 & $0.000^{*}$ & 0.217 \\
\hline Schizoid & $21.3(100)$ & $19.8(67)$ & $25.2(33)$ & 2.32 & 0.128 & 0.073 \\
\hline Avoidant & $19.0(89)$ & $15.4(52)$ & $28.2(37)$ & 11.93 & $0.001 *$ & 0.165 \\
\hline Depressive & $28.1(132)$ & $26.3(89)$ & $32.8(43)$ & 2.91 & 0.88 & 0.082 \\
\hline Dependent & $16.6(78)$ & $13.9(47)$ & $23.7(31)$ & 7.74 & $0.005^{*}$ & 0.133 \\
\hline Histrionic & $0.0(0)$ & $0.0(0)$ & $0.0(0)$ & - & - & - \\
\hline Narcissistic & $11.1(52)$ & $10.1(34)$ & $13.7(18)$ & 1.71 & 0.192 & 0.063 \\
\hline Antisocial & $19.0(89)$ & $19.2(65)$ & $18.3(24)$ & 0.001 & 0.981 & 0.001 \\
\hline Sadistic & $8.1(38)$ & $7.1(24)$ & $10.7(14)$ & 2.02 & 0.156 & 0.068 \\
\hline Compulsive & $0.4(2)$ & $0.0(0)$ & $1.5(2)$ & - & $0.073^{\mathrm{a}}$ & 0.111 \\
\hline Negativistic & $20.7(97)$ & $18.9(64)$ & $25.2(33)$ & 3.06 & 0.080 & 0.084 \\
\hline Masochistic & $22.4(105)$ & $13.9(47)$ & $44.3(58)$ & 55.62 & $0.000^{*}$ & 0.357 \\
\hline Schizotypal & $14.3(67)$ & $11.5(39)$ & $21.4(28)$ & 8.70 & $0.003 *$ & 0.141 \\
\hline Borderline & $20.5(96)$ & $17.2(58)$ & $29.0(38)$ & 9.77 & $0.002^{*}$ & 0.150 \\
\hline Paranoid & $19.4(91)$ & $16.3(55)$ & $27.5(36)$ & 9.10 & $0.003^{*}$ & 0.144 \\
\hline \multicolumn{7}{|l|}{ Clinical Syndromes } \\
\hline Anxiety & $36.2(170)$ & $37.0(125)$ & $34.4(45)$ & 0.15 & 0.701 & 0.018 \\
\hline Somatoform & $8.3(39)$ & $6.2(21)$ & $13.7(18)$ & 7.91 & $0.005^{*}$ & 0.135 \\
\hline Mood disorders & $24.5(115)$ & $20.4(69)$ & $35.1(46)$ & 13.24 & $0.000^{*}$ & 0.174 \\
\hline Problematic alcohol use & $55.9(262)$ & $59.5(201)$ & $46.6(61)$ & 6.55 & $0.010^{*}$ & 0.118 \\
\hline Drug dependence & $34.8(163)$ & $34.6(117)$ & $35.11(46)$ & 0.01 & 0.919 & 0.005 \\
\hline PTSD & $16.4(77)$ & $13.9(47)$ & $22.9(30)$ & 6.71 & $0.010^{*}$ & 0.124 \\
\hline Psychotic disorders & $18.1(85)$ & $13.9(47)$ & $29.0(38)$ & 16.65 & $0.000 *$ & 0.195 \\
\hline Eating disorders & $19.6(92)$ & $15.1(51)$ & $31.3(41)$ & 14.95 & $0.000 *$ & 0.180 \\
\hline Risk of suicidal behaviours & $27.3(128)$ & $23.1(78)$ & $38.2(50)$ & 10.71 & $0.001 *$ & 0.151 \\
\hline
\end{tabular}

*Significant $p<0.05$

${ }^{a}$ Fisher's exact used due to low cell count 
Table 4 Percentage of participants who screened positive for a current mental health need who also report contact with mental health services

\begin{tabular}{|c|c|c|c|c|c|c|}
\hline \multirow[t]{2}{*}{ Disorder } & \multicolumn{3}{|c|}{ Contact mental health services } & \multirow[t]{2}{*}{$\chi^{2}$} & \multirow[t]{2}{*}{$p$} & \multirow[t]{2}{*}{ Effect size } \\
\hline & Total sample, \% (n) & Male, $\%(n)$ & Female, $\%(n)$ & & & \\
\hline Personality disorder & $33.2(85)$ & $24.1(48)$ & $41.6(37)$ & 4.31 & $0.038 *$ & 0.130 \\
\hline Anxiety & $38.8(66)$ & $35.2(44)$ & $48.9(22)$ & 2.61 & 0.106 & 0.124 \\
\hline Somatoform & 48.7 (19) & $42.9(9)$ & $55.6(10)$ & 0.63 & 0.43 & 0.127 \\
\hline Mood disorders & $41.7(48)$ & $36.2(25)$ & $50.0(23)$ & 2.15 & 0.142 & 0.137 \\
\hline Problematic alcohol use & $25.3(66)$ & $21.9(44)$ & $36.7(22)$ & 5.34 & $0.021 *$ & 0.143 \\
\hline Drug dependence & $34.6(56)$ & $29.1(34)$ & $48.9(22)$ & 5.65 & $0.017 *$ & 0.187 \\
\hline PTSD & $54.5(42)$ & $44.7(21)$ & $70.0(21)$ & 4.74 & $0.030^{*}$ & 0.248 \\
\hline Psychotic disorders & $45.9(39)$ & $38.3(18)$ & $55.3(21)$ & 2.44 & 0.119 & 0.169 \\
\hline Eating disorders & $37.4(34)$ & $27.5(14)$ & $50.0(20)$ & 4.87 & $0.027 *$ & 0.231 \\
\hline Risk of suicidal behaviours & $49.6(63)$ & $41.0(32)$ & $63.3(31)$ & 5.95 & $0.015^{*}$ & 0.217 \\
\hline
\end{tabular}

*Significant $p<0.05$

disorder. Males were significantly more likely than females to report having received a previous diagnosis of ADHD. Table 2 shows the prevalence of reported life time diagnosis of mental disorder by disorder type and gender.

\section{Current mental disorder}

\section{Contact with mental health services}

Of the total sample, around a quarter of participants reported that they were currently in contact with mental health services in prison (including Mental Health Inreach and the Offender Personality Disorder Pathway) (25.4\%, $n=119)$. Of those who had reported having received a previous diagnosis of a mental disorder around half also reported currently being in contact with mental health services $(49.7 \%, n=99)$. Females were significantly more likely than males to report having current contact with mental health services, $\chi^{2}(1, N=467)=14.15, p=0.000$.

\section{Screening measure outcomes}

Table 3 details the point-prevalence for each mental disorder screened for by gender, including significant differences. Around two-thirds of participants screened positive for clinical symptoms of at least one type of mental disorder (i.e., above the clinical cut-off) $(66.7 \%, n=313)$. Just over half of all participants screened positive for at least one type of personality disorder $(54.8 \%, n=257)$. The most prevalent personality disorders screened positive for were depressive $(28.1 \%, n=132)$, masochistic $(22.4 \%, n=105)$, and schizoid $(21.3 \%, n=100)$. In terms of other mental health issues, the most common clinical syndromes screened positive for were substance dependence $(42.2 \%, n=198)$, anxiety $(36.2 \%$, $n=170)$, and risk of suicidal behaviour $(27.3 \%, n=128)$.
Females were significantly more likely than males to screen positive for personality disorder; particularly avoidant, dependent, masochistic, schizotypal, borderline, and paranoid personality types. Females were also significantly more likely than males to screen positive for somatoform, mood disorders, PTSD, psychotic disorders, eating disorders, and risk of suicidal behaviour.

With regard to drug dependence, 66.1\% $(n=310)$ of participants reported having used illicit substances prior to coming to prison. Men were significantly more likely than women to report having used drugs in the year prior to prison, $\chi^{2}(1, N=469)=10.06, p=0.002$. The most commonly reported drugs of preference were cannabis $(53.2 \%$, $n=165)$, cocaine $(22.6 \%, n=70)$, and heroin and crack combined $(19.4 \%, n=60)$. Women were significantly more likely than men to report heroin and crack as their combined drug of preference, $\chi^{2}(1, N=309)=34.63, p=0.000$, whereas men were significantly more likely than women to report cannabis, $\chi^{2}(1, N=310)=3.90, p=0.048$, or cocaine, $\chi^{2}(1, N=310)=8.87, p=0.003$, as their drug of preference. Just over a third of participants screened positive for drug dependence $(52.6 \%$ of those who reported using drugs). There was no difference in the percentage of men and women who screened positive for drug dependence. With regard to alcohol use, $55.9 \%(n=262)$ of participants screened positive for increasing/problematic alcohol consumption in the year prior to prison, including $33 \%(n=155)$ for problem drinking, $9.2 \%(n=43)$ for alcohol use disorders, and $13.6 \%(n=64)$ for alcohol dependence. Men were significantly more likely than women to screen positive for some type of problematic alcohol use, $\chi^{2}(1, N=468)=6.55$, $p=0.010$.

Overall $27.3 \%(n=128)$ of participants scored above the cut off for risk of suicidal behaviours. Significantly more women scored above the cut off for risk of suicidal 
behaviours than men, $\chi^{2}(1, N=468)=10.71, p=0.001$. Just over a quarter of participants reported having attempted suicide at least once in their life time $(27.8 \%, n=130)$ and $18.6 \%(n=87)$ of participants reported that they had thought about killing themselves more than once in the past year. Further, $6.9 \%(n=32)$ thought it was likely that they would attempt suicide in the future.

\section{Comorbidity}

Just under half of participants $(47.5 \%, n=223)$ screened positive for two or more types of disorder, with participants screening positive on average for 2.07 disorders (SD 2.24). Women demonstrated significantly higher rates of comorbidity than men, $\chi^{2}(1, N=469)=16.50, p=0.000$ and screened positive for significantly more disorders overall than men, $t(450)=-3.81, p=0.000, d=0.42$.

\section{Met and unmet mental health need}

How well current mental health need was being met for each disorder was calculated using cross-tabs (see Table 4). Met need was low across all disorders screened for. Excluding substance dependence (which is not directly treated by mental health services in the majority of prisons) the disorders with the lowest levels of met need were personality disorder, eating disorders and anxiety. Women's current mental health needs were generally better met than men's, with a higher proportion of participants with identified mental health needs currently reporting contact with prison mental health services. Males were significantly more likely than females to have unmet need in the areas of personality disorder, problematic alcohol use, drug dependence, PTSD, eating disorders and risk of suicidal behaviour.

\section{Discussion}

This study provides an updated picture of the mental health needs of both male and female prisoners in the UK. A crosssection of male and female prisoners from 13 prisons in one region of the UK were assessed for a range of mental health issues using a battery of validated screening measures. The current study represents one of the largest studies of the mental health needs of prisoners in the UK, in terms of sample size and number of establishments included, since the ONS Survey in 1997 [4]. Over half of participants reported having had previous contact with mental health services either in prison or in the community, rates much higher than those reported in the general population [21]. Further a high number of participants reported having previously being diagnosed with a mental disorder; however, only around half of these also reported current contact with prison mental health services. In terms of current mental health need, prevalence rates for current mental disorder were high, with particularly high levels noted for personality disorder, anxiety, mood disorders and risk of suicidal behaviours. Levels of comorbidity were high, with over half of all participants screening positive for two or more types of mental disorder on the screening measures. Of those participants who screened positive for a current mental health issue, a large proportion reported having no current contact with mental health services. Females reported significantly higher levels of mental health need compared to males (both current and pre-existing): particularly in relation to personality disorders, mood disorders, PTSD, eating disorders, psychotic disorders, and risk of suicidal behaviours. Females' mental health needs appeared to be better met than that of males.

\section{Comparisons with the literature}

Rates of previous contact with mental health services and having a lifetime diagnosis of a mental disorder are similar to those reported in previous research with UK prison samples $[5,7]$, indicating that a significant proportion of prisoners continue to arrive in prison with pre-existing mental health issues. Despite this, less than half of participants with a pre-existing diagnosis reported current contact with Mental Health Inreach. This finding is consistent with previous research [22], suggesting that only a relatively low proportion of individuals who arrive in prison with pre-existing mental health issues are both identified and provided with treatment.

Rates of mental disorder in the current study were well above those of the general population reported in meta-analyses and large population studies, consistent with previous research in the area [4-6], suggesting that mental disorder continues to be highly prevalent amongst individuals in prisons. In particular, prevalence rates were 4.5-5 times higher than that found in the general population for personality disorder (54.8\% vs. $12.2 \%$ [23]), anxiety (36.2\% vs. $6.7 \%$ [24]), mood disorders (24.5\% vs. 5.4\% [24]), and PTSD (16.4\% vs. $2 \%$; [25]). Prevalence rates were also above that of the general population for both psychotic disorders (18.1\% vs. $0.03 \%$ [26]), and eating disorders (19.6\% vs. $10.1 \%$ [27]). The elevated rates of unmet need detected in the current study are consistent with those found in previous research [6], suggesting that many individuals in prison experiencing current mental health issues either go unidentified or are unable to access treatment.

Prevalence rates for both pre-existing and current mental health needs were generally higher among women compared to men, consistent with previous research [4-7, 28, 29]. Prevalence rates across the range of mental disorders screened for varied slightly to other previous UK-based 
research in the area [5, 6]. For example, rates for drug and alcohol dependence were much lower and rates of anxiety were slightly higher. These differences may reflect changes in the prison population (e.g., overall increase) or variations in the types of prisoners sampled (e.g., different proportions of remand and sentenced prisoners). Alternatively, this may reflect differences in the measures used to assess mental disorder across studies. For example, the current study utilised self-report screening measures, as opposed to diagnostic measures or structured clinical interviews. Some researchers have argued that screening measures can sometimes overestimate the prevalence of disorders in prison samples due to the inclusion of non-specific items and lack of validation in this population [1]. However, the measures used in the current study are frequently used for screening in forensic settings as well as in prison research. Further, given the current study used a cross-sectional design it is also possible that some individuals with mental health issues were either not captured or declined to take part in the study.

One notable point is that this study is one of the first to examine the prevalence of eating disorders in both male and female UK prisoners using a standardised screening measure. Previous research in this area has either collected selfreport data on previous diagnosis of an eating disorder [5] or has conducted screenings with just a small sample of women in a single establishment [20]. The prevalence rates of risk of eating disorders among women in the current sample were 2.5 times higher than the rate detected in the general population using the same measure [13], with the reported rate amongst men being just above that of the general population. This suggests that women in prison may be at a higher risk of eating issues compared to the general population.

\section{Generalisability}

Whilst this study represents one of the largest epidemiological studies of mental disorder in prisoners undertaken in the UK since the ONS 1997 survey, the sample size is still relatively small when compared to the total UK prison population (3.4\% female population, $0.4 \%$ male population). However, the prisons that the study sample was drawn from represent a third of all female establishments and approximately $10 \%$ of all adult male establishments in the UK. Further, the findings of the current research represent individuals from across a range of prison settings (high security, closed conditions, open conditions, local remand), sentence lengths (short and long term), and offence types. Although the current study draws upon a wide and diverse sample of participants across a range of settings, some groups were under-represented in the sample. For example, older adults (i.e., those over 55 years old) represented only a small proportion of the overall sample (7.9\%) as did those on remand $(9.8 \%)$. The under-representation of these groups can be partly attributed to the establishments in the region from where participants were recruited from (i.e., the majority holding sentenced prisoners). However, given that previous research has shown that remand prisoners have slightly differing mental health needs to sentenced prisoners and that the number of older adults detained in prisons has rapidly increased in recent years, further work is needed to understand the specific mental health, social care and treatment needs of these populations to inform policy and provision for these groups [1].

It is important to consider, when interpreting the results of this study, that the findings represent a point-in-time snapshot; thus, it provides an updated picture of the mental health needs of men and women detained in UK prisons sampled at the time of the research. However, any conclusions regarding causality of mental ill-health are not able to be drawn from the findings. Previous research shows that compared to the general population, people in prison are significantly more likely to have experienced adverse events prior to incarceration which have been linked to the development of mental health issues. Thus, many people in prison experience mental health issues prior to their arrival in custody [30]. For example, "imported factors" such as pre-prison dispositions, childhood sexual abuse, and learning difficulties have been found to be significant predictors of within-prison mental health status [31]. However, the psychosocial experience of imprisonment has also been found to act as an additional stressor upon individuals' mental wellbeing [32]. Subsequently, there is a wider need for high-quality longitudinal research to examine the impact of the current prison environment on mental health, risk factors for the development of mental health issues in prison, and the effectiveness of interventions for assisting individuals in prison to recover/ manage their mental health needs. Such research should also focus on current use of substances, including psychoactive substances, within the prison environment and self-injurious behaviours, which were not examined as part of the current study.

\section{Clinical implications and future directions}

Identification of both pre-existing and current mental health issues amongst those detained in prisons is critical for the planning and development of assessment and treatment services in these settings, as well as being vital for helping those who need it to access appropriate support both within the prison environment, with legal proceedings, and for successful rehabilitation and community reintegration [33]. However, both the current study and previous research in the field suggest that only a small proportion of individuals who either report a previous diagnosis or screen positive for a current mental health need currently receive treatment within prison $[6,22]$; potentially indicating high levels of 
unmet need. Previous research suggests that as many as $46-64 \%$ of prisoners' mental health needs remain unmet $[6$, $22]$, with the current study highlighting significant differences in the levels of unmet need between men and women across several mental disorders. Thus, accurate and timely identification of treatment needs is critical to improving this rate. To aid this task, it is essential that community mental health services, general practitioners, and prison Mental Health Inreach teams work together to ensure continuity of care during the transition process between community and custody and vice versa. Joint working and the sharing of information between services will not only enable more effective identification of individuals with known mental health issues but will also enable effective and efficient continuity of treatment.

In terms of identifying mental health needs upon individuals' arrival in prison, many factors may impact upon the initial screening process, whether someone is accepted for treatment, and how long the wait for this is. However, our findings reinforce previous research, suggesting that current screening tools potentially lack sensitivity and specificity [22] and that a more comprehensive, in-depth, and genderfocused primary mental health screen upon initial reception to prison could help to identify those who warrant further assessment and/or treatment for mental health issues; for example, incorporating standardised screening for eating disorders, suicidal risk and PTSD for female prisoners. Further, initial reception screening alone may not be sufficient to identify all of those who have or may develop mental health issues as a result of incarceration. The current study sampled prisoners at various stages in their sentence pathway and the high levels of prevalence detected suggests that additional screening points during incarceration may be beneficial to ensure adequate support is provided as and when needed. Research examining the effectiveness of current screening and care models in identifying and meeting the mental health needs of those in prison would be highly beneficial to healthcare providers, commissioners, and policy makers.

\section{Conclusions}

Pre-existing and current mental health issues remain highly prevalent amongst individuals detained in prison in the UK. Prevalence rates appear to be relatively similar to those reported 20 years ago; however, the prison population has dramatically increased during this time. High levels of comorbidity were found in the current study, with a significant proportion of prisoners screening positive for two or more disorders. However, only around half of those with a pre-existing diagnosis of a mental disorder were currently receiving treatment from prison mental health services and levels of unmet current mental health need were high. In addition, females appear to have higher levels of overall mental health need compared to males across a range of disorders, although these appear to be generally better met in terms of contact with services. Whilst this study is crosssectional in design, its findings can help to inform the development and delivery of care models and health initiatives in UK prisons. The recent NAO report [2] highlights the current weaknesses in identifying those with mental health needs. Both the NAO report and the recent NICE guidelines [3] provide a clear set of recommendations to enable the effective identification, coordination, and delivery of care. However, in order to effectively deliver these investment is required to improve prison staff knowledge and understanding of mental illness as well as the screening process to ensure the effective identification and treatment of individuals with mental health needs in prison.

\section{Compliance with ethical standards}

Conflict of interest The original project from which this study derived from was funded by NHS England. The funding source had no role in the design and conduct of the study; collection, management, analysis, or interpretation of data; or preparation of the manuscript. At the time of the research NT was employed by the University of Kent and Kent and Medway NHS Partnership Trust; HM was employed by Kent and Medway NHS Partnership Trust, the University of Kent and the Institute of Psychiatry, Psychology and Neuroscience; BK and GR were employed by Kent and Medway NHS Partnership Trust.

Open Access This article is distributed under the terms of the Creative Commons Attribution 4.0 International License (http://creativeco mmons.org/licenses/by/4.0/), which permits unrestricted use, distribution, and reproduction in any medium, provided you give appropriate credit to the original author(s) and the source, provide a link to the Creative Commons license, and indicate if changes were made.

\section{References}

1. Fazel S, Hayes AJ, Bartellas K, Clerici M, Treatman R (2016) The mental health of prisoners: a review of prevalence, adverse outcomes and interventions. Lancet Psychiatry 3:871-881

2. National Audit Office (NAO) (2017) NAO Mental Health in Prisons. NAO. https://www.nao.org.uk/wp-content/uploads/2017/06/ Mental-health-in-prisons.pdf. Accessed 26 Nov 2017

3. National Institute of Health \& Care Excellence (NICE) (2017) NICE Mental Health of Adults in contact with the Criminal Justice System. NICE. https://www.nice.org.uk/guidance/ng66. Accessed 2 Apr 2017

4. Singleton N, Meltzer H, Gatward R, Coid J, Deasy D (1998) Psychiatric morbidity among prisoners in England and Wales: summary report. Office for National Statistics, London

5. Bebbington P, Jakobowitz S, McKenzie N, Killaspy H, Iveson R, Duffield G et al (2017) Assessing needs for psychiatric treatment in prisoners: 1. Prevalence of disorder. Soc Psychiatry Psychiat Epidemiol 52:221-229

6. Jakobowitz S, Bebbington P, McKenzie N, Iveson R, Duffield G, Kerr M et al (2017) Assessing needs for psychiatric treatment in prisoners 2. Met and unmet need. Soc Psychiatry Psychiat Epidemiol 52:231-240 
7. Light M, Grant E, Hopkins K (2013) Gender differences in substance misuse and mental health amongst prisoners: Results from the Surveying Prisoner Crime Reduction (SPCR) longitudinal cohort study of prisoners. Ministry of Justice (MOJ) Analytical Series, 2013

8. Archer RP, Buffington-Vollum JK, Vauter Stredny R, Handel RW (2006) A survey of psychological test use patterns among forensic psychologists. J Pers Assess 87:84-94

9. Milligan JR, Waller G, Andrews B (2002) Eating disturbances in female prisoners: the role of anger. Eat Behav 3:101-204

10. Neal TMS, Grisso T (2014) Assessment practices and expert judgment methods in forensic psychology and psychiatry: an international snapshot. Crim Justice Behav 41(12):1406-1421

11. Strang J, Gossop M, Heuston J, Green J, Whiteley C, Maden A (2006) Persistence of drug use during imprisonment: relationship of drug type, recency of use and severity of dependence to use of heroin, cocaine and amphetamine in prison. Addiction 101(8):1125-1132

12. Maggia $B$, Martin $S$, Crouzet $C$, Richard $P$, Wagner $P$, Balmès J-L, Nalpas B (2004) Variation in AUDIT (Alcohol use disorder identification test) scores within the first weeks of imprisonment. Alcohol 39(3):247-250

13. Millon C, Davis R, Grossman S (2006) MCMI-III: Millon Clinical Multiaxial Inventory-III: Pearson Assessments, 2006

14. Gossop M, Darke S, Griffiths P, Hando J, Powis B, Hull W et al (1995) The severity of dependence scale (SDS): psychometric properties of the SDS in English and Australian samples of heroin, cocaine and amphetamine users. Addiction 90:607-614

15. Piccinelli M, Tessari E, Bortolomasi M, Piasere O, Semenzin M, Garzotto N, Tansella M (1997) Efficacy of the alcohol use disorders identification test as a screening tool for hazardous alcohol intake and related disorders in primary care: a validity study. BMJ 314:420-424

16. Osman A, Kooper AB, Linehan MM, Barrios XF, Gutierrez MP, Bagge LC (1999) Validation of the adult suicidal ideation questionnaire and the reasoms for living inventiory in an adult psychiatric inpatient sample. Psychol Assess 11:115-123

17. Osman A, Bagge CL, Guitierrez PM, Konick LC, Kooper BA, Barrios FX (2001) The suicidal behaviors questionnaire-revised (SBQ-R), Validation with clinical and nonclinical samples. Assessment 5:443-454

18. Morgan JF, Reed F, Lacey JH (1999) The SCOFF questionnaire: assessment of a new screening tool for eating disorders. BMJ 319:1467-1468

19. Sturge G (2018) UK prison population statistics. Briefing Paper, House of Commons Library

20. Office for National Statistics (2018) Reporting on the sustainable development goals: People on remand in custody in England and Wales, 2018
21. Singleton N, Bumpstead R, O’Brien M, Lee A, Meltzer H (2000) Psychiatric morbidity among adults living in private households. TSO, London

22. Senior J, Birmingham L, Harty MA, Hassan L, Hayes AJ, Kendall $\mathrm{K}$ et al (2013) Identification and management of prisoners with severe psychiatric illness by specialist mental health services. Psychol Med 43:1511-1520

23. Volkert J, Gablonski TC, Rabung S (2018) Prevalence of personality disorders in the general adult population in Western countries: systematic review and meta-analysis. Br J Psychiatry 213(6):709-715

24. Steel Z, Marnane C, Iranpour C, Chey T, Jackson JW, Patel V, Silove D (2014) The global prevalence of common mental disorders: a systematic review and meta-analysis 1980-2013. Int J Epidemiol 43:476-493

25. Frans Ö, Rimmő P-A, Aberg L, Fredrikson M (2005) Trauma exposure and post-traumatic stress disorder in the general population. Acta Psychiatr Scand 111:291-299

26. Linscott RJ, van Os J (2013) An updated and conservative systematic review and meta-analysis of epidemiological evidence on psychotic experiences in children and adults: on the pathway from proneness to persistence to dimensional expression across mental disorders. Psychol Med 43(6):1133-1149

27. Solmi F, Hatch SL, Hotopf M, Treasure J, Micali N (2014) Prevalence and correlates of disordered eating in a general population sample: the South East London Community Health (SELCoH) study. Soc Psychiatry Psychiatr Epidemiol 49(8):1335-1346

28. Fazel S, Seewald K (2012) Severe mental illness in 33588 prisoners worldwide: Systematic review and meta-regression analysis. Br J Psychiatry 200:364-373

29. Fazel S, Yoon AI, Hayes JA (2017) Substance use disorders in prisoners: an updated systematic review and meta-regression analysis in recently incarcerated men and women. Addiction 112:1725-1739

30. Harvey J, Smedley K (2010) Psychological therapy in prisons and other secure settings. Routledge, London

31. Bowler N, Phillips C, Rees P (2018) The association between imported factors and prisoners mental health: Implications for adaptation and intervention. Int J Law Psychiatry 57:61-66

32. Leibling A, Maruna S (2005) The effects of imprisonment. Willan Publishing, Cullompton

33. Forrester A, Till A, Simpson A, Shaw J (2018) Mental illness and the provision of mental health services in prison. Br Med Bull 1:101-109 